\title{
Potential Pitfalls of Diaphragm Structural Matching in Carbon-ion Radiotherapy for Pancreatic Cancer
}

\author{
YUSUKE ITABASHI $^{1}$, YOSHIKI KUBOTA ${ }^{2}$, MASAHIKO OKAMOTO ${ }^{2}$, KAZUHISA TSUDA $^{1}$, \\ SHINTARO SHIBA ${ }^{2}$, YOSHIHIKO HOSHINO ${ }^{1}$, TAKAYUKI SUTO ${ }^{1}$, TATSUYA OHNO ${ }^{2}$ and TAKASHI NAKANO ${ }^{2}$ \\ ${ }^{1}$ Department of Radiology, Gunma University Hospital, Gunma, Japan \\ ${ }^{2}$ Gunma University Heavy Ion Medical Center, Gunma, Japan
}

\begin{abstract}
Background/Aim: To evaluate the effectiveness of diaphragm matching (DM) for carbon-ion radiotherapy (CIRT) of pancreatic cancer patients and develop a simple method to estimate tumour position. Patients and Methods: Treatment planning CTs from 27 pancreatic cancer patients treated with CIRT in our facility were used in this study, and 32 other CT image datasets taken on different days were used for measuring tumour and diaphragm displacements. A correction method (SI-correction) was developed using the coefficient $x$ of the regression line formula for the displacements between the diaphragm and tumour in the superior-inferior direction. The tumour positioning errors of bone matching (BM), DM, and SI-correction were measured. Results: Mean ( \pm standard deviation) absolute errors of $B M$, $D M$, and SI-correction were 5.10 $\pm 3.31,7.48 \pm 4.04$, and $4.13 \pm 2.51 \mathrm{~mm}$, respectively. DM showed significant differences compared to the other correction methods. Conclusion: DM was subject to larger errors than BM. Our correction method improved positional errors.
\end{abstract}

Pancreatic cancer is difficult to detect at an early stage and has a low survival rate compared to other cancers (1). At the time of diagnosis, patients often have metastatic or locally advanced unresectable disease. Chemotherapy or chemoradiotherapy are considered standard treatments for locally advanced pancreatic cancer (2). Kawashiro et al. have reported that carbon-ion radiotherapy (CIRT) improved the overall survival rate compared to

This article is freely accessible online.

Correspondence to: Yoshiki Kubota, Ph.D., Gunma University Heavy Ion Medical Center, 3-39-22 Showa-machi, Maebashi, Gunma 371-8511, Japan. Tel: +81 272208378, Fax: +81 272208379, e-mail: y_kubota@gunma-u.ac.jp

Key Words: Carbon ion radiotherapy, pancreatic cancer, patient positioning, diaphragm matching, bone matching. chemotherapy and chemoradiotherapy (3). Therefore, CIRT is considered a promising new therapy for locally advanced pancreatic cancer.

Carbon-ion beams have the advantage of a higher dose concentration than photon beams (4). However, there is the risk that the dose distribution may be substantially changed from the treatment planning dose distribution if the target position is shifted or the water equivalent path length (WEL) to the target is changed, and pancreatic cancer dose distributions are particularly affected by WEL change. In particular, the reproducibility of the dose distribution may be greatly reduced in mobile organs (5-9), although tumour matching (TM) can improve dose reproducibility (9).

However, there are not many CIRT facilities in which an Xray $\mathrm{CT}$ system is positioned in the irradiation room, because of the problem of interference with the irradiation device, and therefore, orthogonal X-ray images are generally used for patient positioning (10-12). Thus, with such facilities, it is difficult to confirm the position of soft tissue, such as pancreatic tumour, during patient positioning for irradiation. In addition, even if an X-ray CT system is installed, it is not possible to acquire real-time CT images during irradiation. To improve the accuracy of the carbon-ion beam irradiation to the target, it would be desirable to confirm the tumour position on the X-ray images acquired during the treatment. A metal marker visible on the X-ray image can be inserted near or in the tumour, and this is helpful for estimating the tumour location. In pancreatic cancer, a metal marker may be inserted under endoscopic ultrasound, but this is not yet a generally performed procedure. Thus, another index that can estimate the tumour position on Xray images without the use of a metal marker is necessary. It is considered possible to estimate the tumour position according to diaphragm movement, because the diaphragm moves with patient's respiration. However, there is no evidence demonstrating the correlation between diaphragm displacements and tumour displacements in pancreas.

Therefore, we used CT images to evaluate the correlation between the diaphragm displacement and tumour displacement, and examined whether diaphragm matching (DM) can be 
effective for estimating tumour position in a pancreatic cancer patient. Furthermore, we also developed and evaluated a new method to estimate tumour position.

\section{Patients and Methods}

Patient selection. Data from patients with pancreatic cancer who were treated with CIRT using the passive irradiation method at Gunma University Heavy Ion Medical Center were used in this study. The patients' characteristics are shown in Table I. This study was approved by the Institutional Review Board at Gunma University Hospital (number: 2018-095), and was performed in accordance with the ethical guidelines and regulations. All patient data were anonymized.

CT image acquisition. Treatment planning CT (Plan-CT) images were acquired on an Acquilion LB scanner (Self-Propelled, Canon Medical Systems, Japan) around expiration phase. A respiratory gating system (AZ-733, Anzai Medical, Japan) was used to monitor patient respiratory motion. CT images were also acquired on a different day to the Plan-CT, to confirm the reproducibility of tissue identification inside the patient and the dose distributions (ConfCT). Twenty-eight sets of Plan-CT images and 34 sets of Conf-CT images were used in this study. The mean time interval between the Plan-CT and Conf-CT was 13.5 days (range $=10-36$ days).

Correction method. Displacements from the bone matching (BM) to $\mathrm{DM}$ positions and from the $\mathrm{BM}$ to $\mathrm{TM}$ positions were measured in the Plan-CT and Conf-CT, respectively. For the DM, commercial registration software (MIM Maestro, MIM Software, USA) was used to register the diaphragm tip to the BM position, using only translation in three directions: right-left (R-L), anterior-posterior (A-P), and superior-inferior (S-I). Sample images for the BM and DM are shown in Figure 1.

To estimate the tumour position, correlations and regressions between the diaphragm displacements and tumour displacements, calculated from the BM position, were determined. The estimated position of the tumour was calculated by multiplying the coefficient $\mathrm{x}$ of the regression line formula by the diaphragm displacements. Two correction methods were applied, a 3D-correction involving correction of the values of all diaphragm displacements in the $\mathrm{R}-\mathrm{L}$, $\mathrm{A}-\mathrm{P}$, and S-I directions, and an SI-correction involving correction of the diaphragm displacements in only the S-I direction, with the $\mathrm{R}-\mathrm{L}$ and A-P positions being taken from the BM. Because of the low number of CT image sets, a cross validation procedure was used, with one CT image set being used to evaluate the correction positions separately for the pancreatic head and body, while the other CT image sets were used to calculate the regression line. This procedure was repeated using each of the $\mathrm{CT}$ image sets as the evaluation set.

Evaluation method. Positional errors for the BM, DM, 3D-correction, and SI-correction were defined by calculating the Euclidean distance between each position and tumour position. The Bonferroni method was used to correct for multiple comparisons between the positional errors of BM, DM, 3D-correction, and SI-correction after testing for normality with the Shapiro-Wilk test. A level of $p<0.05$ was considered statistically significant. All statistical analyses were performed using SPSS software (IBM SPSS Statistics for Windows, version 25.0, IBM, Inc., Armonk, NY, USA).
Table I. Patient characteristics.

\begin{tabular}{lc}
\hline Number of patients (CT image sets) & $27(32)$ \\
Age & $52-88$ (median 70) \\
Gender (male/female) & $15 / 12$ \\
Tumour location (head/body) & $10 / 17$ \\
Tumour volume (ml) & $14 / 18$ \\
Number of CT image sets (head/body) & $2.38-56.96$ (median 12.49$)$ \\
\hline
\end{tabular}

\section{Results}

The tumour and diaphragm displacements are shown in Table II, and correlations between the tumour displacements and diaphragm displacements are shown in Figure 2. The mean \pm standard deviation of the coefficient $x$ of the regression lines (correlation coefficient $\mathrm{R}$ ) for the pancreatic body were $0.66 \pm 0.17,0.17 \pm 0.04$, and $0.39 \pm 0.02(0.42 \pm 0.05$, $0.40 \pm 0.07$, and $0.93 \pm 0.01)$ in R-L, A-P, and S-I directions, respectively, while for the pancreatic head they were $0.87 \pm 0.11,0.05 \pm 0.03$, and $0.41 \pm 0.02(0.41 \pm 0.04,0.12 \pm 0.08$, and $0.76 \pm 0.04)$, respectively. There were strong correlations in both the pancreatic head and body in the S-I direction, but only weak correlations in the $\mathrm{R}-\mathrm{L}$ and $\mathrm{A}-\mathrm{P}$ directions. The displacement errors for BM, DM, 3D-correction, and SIcorrection are shown in Table III. There were significant differences between DM errors and those of BM, 3Dcorrection, and SI-correction in the total of the pancreas, but there were no significant differences in errors between BM, 3D-correction, and SI-correction.

\section{Discussion}

In this study, we evaluated the effectiveness of DM for determining tumour displacements for CIRT for pancreatic cancer, and developed a new method to estimate tumour position using the equation for the regression line between the tumour and diaphragm displacements. Table II shows that the inter-fraction tumour displacements were larger than those reported in Akimoto et al. or Whitfield et al. $(13,14)$, while diaphragm displacements were larger than the tumour displacements. There were strong correlations between tumour displacements and diaphragm displacements in the $\mathrm{S}-\mathrm{I}$ direction, as shown in Figure 2.

Although there was a strong correlation, the positional errors with DM were significantly larger than those with $\mathrm{BM}$, because the amplitudes of the tumour displacement and diaphragm displacements differed substantially (Table III). Thus, DM is recommended as an index for determining if the tumour has moved, but it is not recommended as a surrogate measure of tumour position for radiotherapy. Although the positional errors with BM were smaller than 

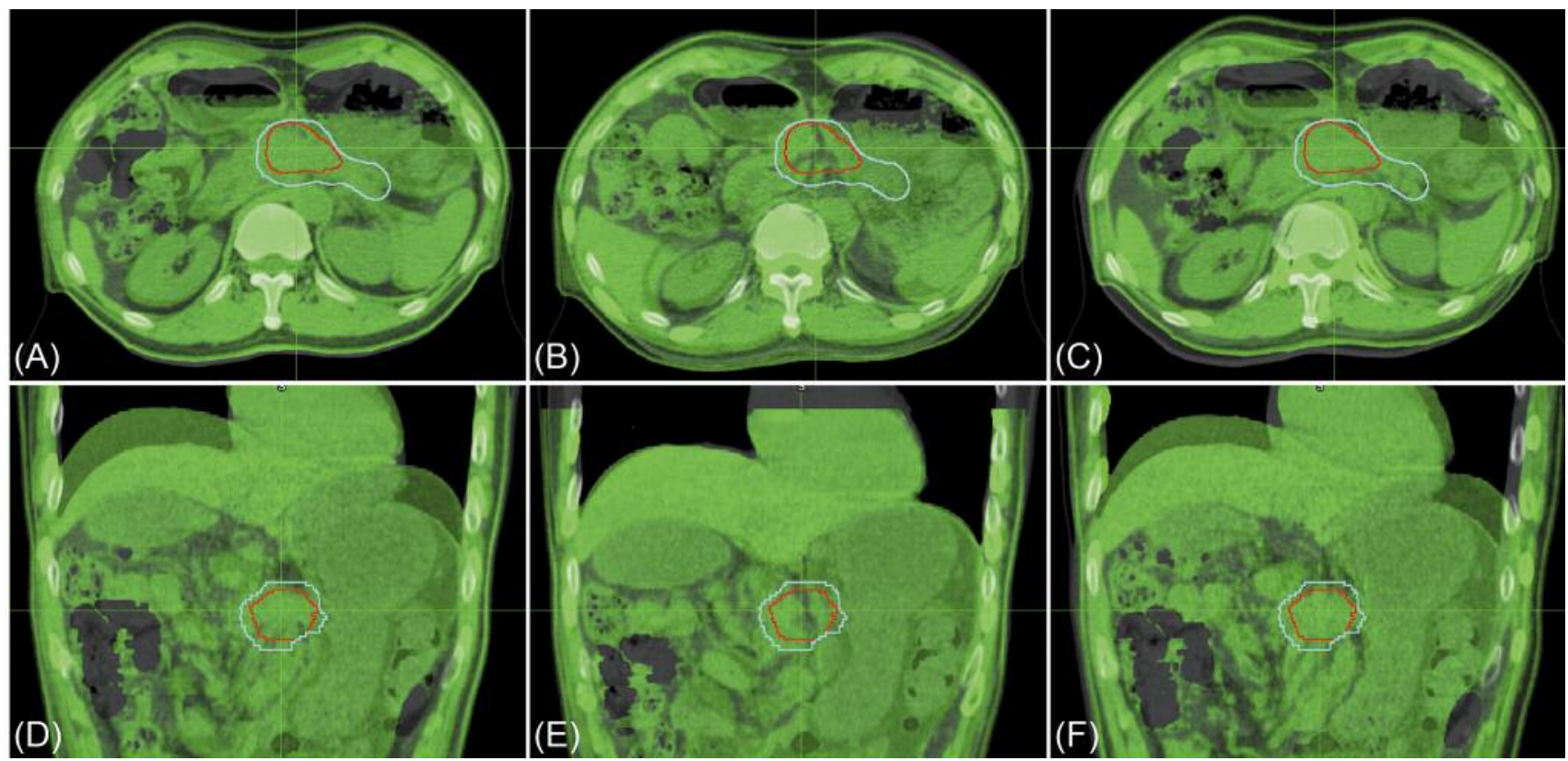

Figure 1. Overlay of the Plan-CT and Conf-CT images. $(A, D)$ Registered with $B M,(B, E)$ registered with DM, $(C, F)$ registered with TM. $(A, B, C)$ show axial images, and $(D, E, F)$ show coronal images. The plan-CT images are displayed in grey-scale and the Conf-CT images are displayed in a green-wash colour. The gross tumour volume (GTV) and clinical target volume (CTV) are outlined in red and cyan, respectively.

Table II. Tumour and diaphragm displacements $(n=32)$. All numbers represent the average \pm standard deviation.

\begin{tabular}{|c|c|c|c|c|c|c|}
\hline & \multicolumn{2}{|c|}{ Head (mm) } & \multicolumn{2}{|c|}{ Body (mm) } & \multicolumn{2}{|c|}{ Total (mm) } \\
\hline & $\begin{array}{c}\text { Tumour } \\
\text { displacement }\end{array}$ & $\begin{array}{c}\text { Diaphragm } \\
\text { displacement }\end{array}$ & $\begin{array}{c}\text { Tumour } \\
\text { displacement }\end{array}$ & $\begin{array}{c}\text { Diaphragm } \\
\text { displacement }\end{array}$ & $\begin{array}{c}\text { Tumour } \\
\text { displacement }\end{array}$ & $\begin{array}{c}\text { Diaphragm } \\
\text { displacement }\end{array}$ \\
\hline RL & $\begin{array}{c}-0.29 \pm 3.56 \\
(-7.62 \text { to } 5.68)\end{array}$ & $\begin{array}{c}-0.87 \pm 2.31 \\
(-6.46 \text { to } 1.92)\end{array}$ & $\begin{array}{c}-0.77 \pm 3.50 \\
(-8.75 \text { to } 7.81)\end{array}$ & $\begin{array}{c}-0.27 \pm 1.49 \\
(-3.48 \text { to } 2.21)\end{array}$ & $\begin{array}{c}-0.56 \pm 3.33 \\
(-8.75 \text { to } 7.81)\end{array}$ & $\begin{array}{c}-0.54 \pm 1.88 \\
(-6.46 \text { to } 2.21)\end{array}$ \\
\hline AP & $\begin{array}{c}-0.63 \pm 2.31 \\
(-4.84 \text { to } 2.89)\end{array}$ & $\begin{array}{c}-0.68 \pm 5.34 \\
(-8.16 \text { to } 10.39)\end{array}$ & $\begin{array}{c}0.12 \pm 1.56 \\
(-2.91 \text { to } 2.09)\end{array}$ & $\begin{array}{c}0.14 \pm 3.65 \\
(-10.29 \text { to } 6.42)\end{array}$ & $\begin{array}{c}-0.21 \pm 1.93 \\
(-4.84 \text { to } 2.89)\end{array}$ & $\begin{array}{c}0.14 \pm 3.65 \\
(-10.29 \text { to } 6.42)\end{array}$ \\
\hline SI & $\begin{array}{c}-1.61 \pm 4.29 \\
(-6.81 \text { to } 9.55)\end{array}$ & $\begin{array}{c}-4.68 \pm 10.12 \\
(-20.38 \text { to } 16.31)\end{array}$ & $\begin{array}{c}-0.40 \pm 4.99 \\
(-10.52 \text { to } 8.94)\end{array}$ & $\begin{array}{c}1.91 \pm 9.26 \\
(-17.80 \text { to } 21.03)\end{array}$ & $\begin{array}{c}-0.93 \pm 4.66 \\
(-10.52 \text { to } 9.55)\end{array}$ & $\begin{array}{c}1.91 \pm 9.26 \\
(-17.80 \text { to } 21.03)\end{array}$ \\
\hline Absolute value & $\begin{array}{c}5.48 \pm 2.71 \\
(1.91 \text { to } 12.55)\end{array}$ & $\begin{array}{c}10.53 \pm 6.47 \\
(0.42 \text { to } 21.17)\end{array}$ & $\begin{array}{c}4.80 \pm 3.76 \\
(0.57 \text { to } 11.15)\end{array}$ & $\begin{array}{c}8.40 \pm 6.04 \\
(2.75 \text { to } 21.99)\end{array}$ & $\begin{array}{c}5.10 \pm 3.31 \\
(0.57 \text { to } 12.55)\end{array}$ & $\begin{array}{c}9.16 \pm 6.23 \\
(0.42 \text { to } 21.99)\end{array}$ \\
\hline
\end{tabular}

RL: Right-left; AP: anterior-posterior; SI: superior-inferior; Head: pancreatic head; Body: pancreatic body; Total: both pancreatic head and body.

those with DM, BM was not accurate enough, because it still had errors of $5.10 \pm 3.31 \mathrm{~mm}$. The positional errors of our proposed 3D-correction and SI-correction methods were smaller than those of BM in both the pancreatic head and body, although the differences were not statistically significant. There were no large differences between the errors of the 3D-correction and SI-correction, which led us to assume that the diaphragm displacements in the $\mathrm{R}-\mathrm{L}$ and A-P directions were not useful for estimating tumour position because the correlations were low. Thus, SIcorrection might be more suitable for a clinical site, because it requires a simple movement in only the S-I direction. Comparison of the SI-correction errors in the pancreatic head with those in the pancreatic body revealed that the pancreatic head showed lower errors. We assumed that the correction is more effective in the pancreatic head than in the pancreatic body, because it has a higher correlation $(\mathrm{R}=0.93 \pm 0.01)(\mathrm{R}=0.76 \pm 0.04)$, although we also 
(A)

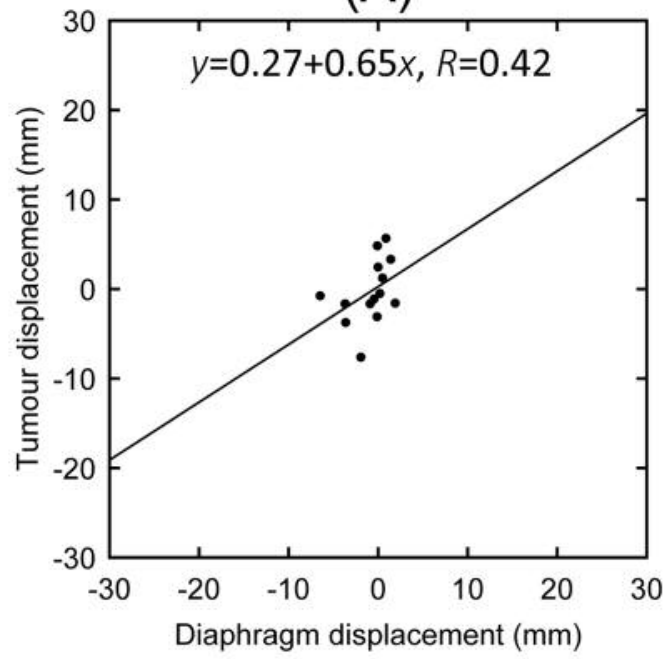

(C)

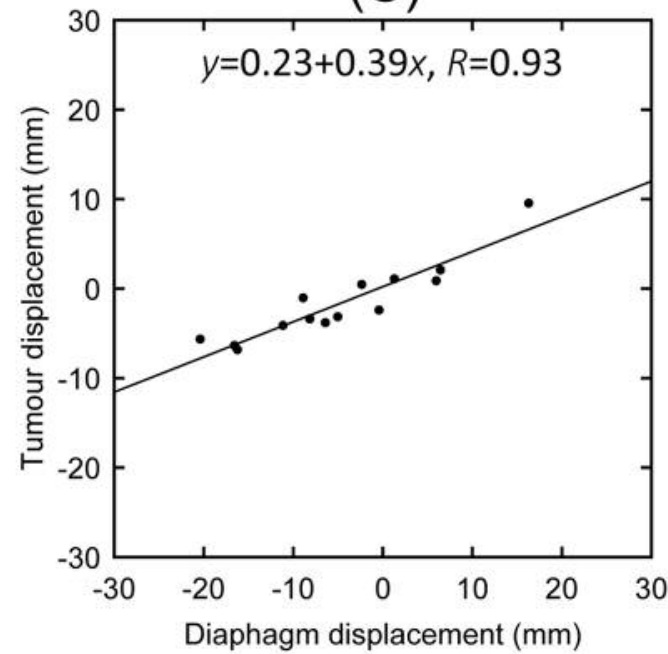

$(\mathrm{E})$

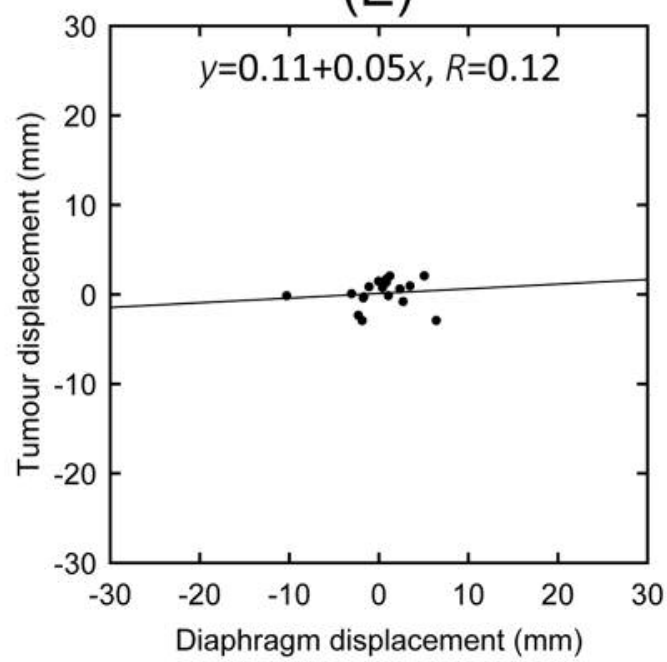

(B)

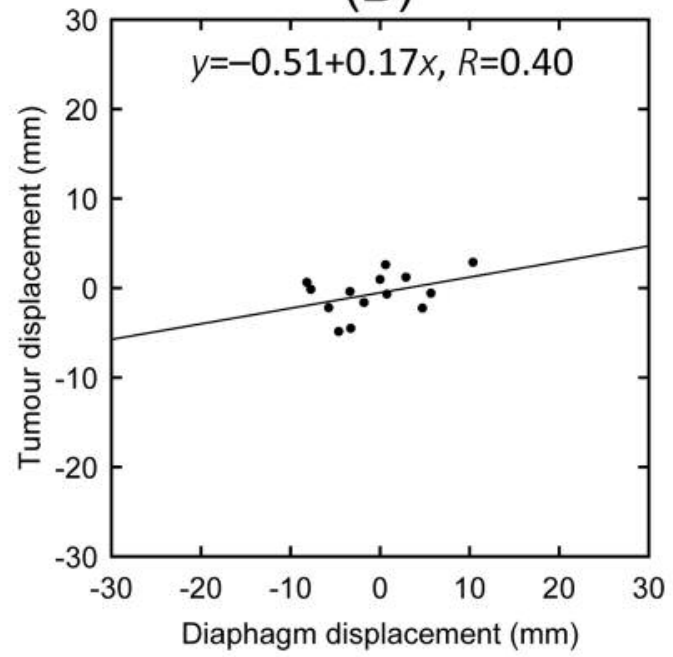

(D)

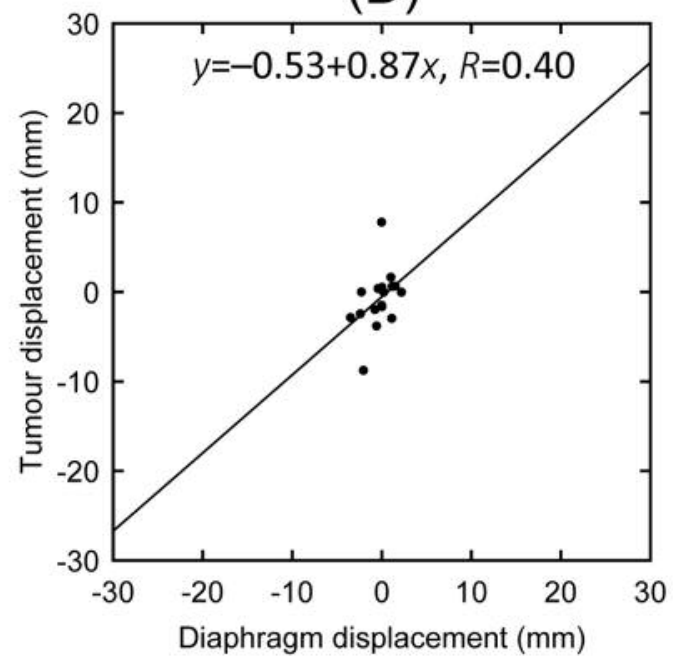

(F)

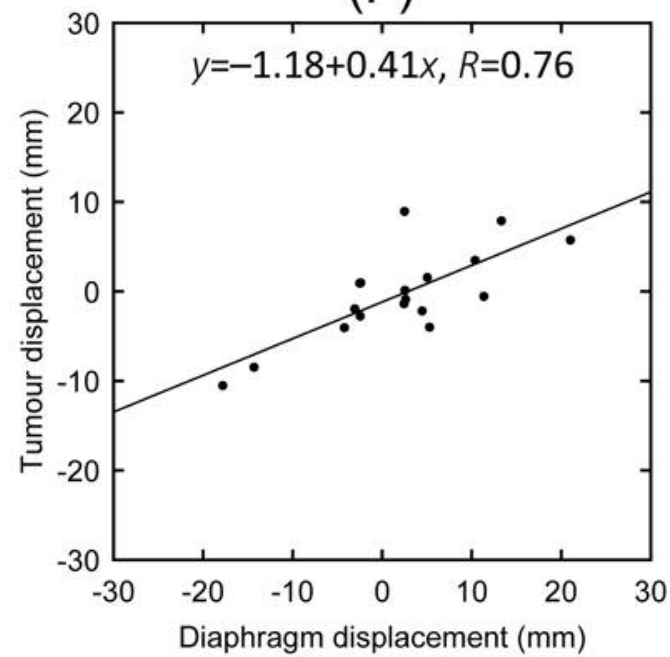

Figure 2. Correlations between diaphragm displacement and tumour displacement from bone matching positioning. $(A, D) R-L$ direction, $(B, E)$ $A-P$ direction, and $(C, F) S-I$ direction. $(A, B, C)$ pancreatic head and $(D, E, F)$ pancreatic body. 
Table III. Difference between tumour position and corrected position using diaphragm displacement $(n=32)$. All numbers represent the average \pm standard deviation (range) of absolute displacements.

\begin{tabular}{lccc}
\hline & Head $(\mathrm{mm})$ & Body $(\mathrm{mm})$ & Total $(\mathrm{mm})$ \\
\hline BM & $5.48 \pm 2.71$ & $4.80 \pm 3.76$ & $5.10 \pm 3.31^{*}$ \\
& $(1.91$ to 12.55$)$ & $(0.57$ to 11.15$)$ & $(0.57$ to 12.55$)$ \\
DM & $8.04 \pm 4.05$ & $7.05 \pm 4.10$ & $7.48 \pm 4.04$ \\
& $(1.85$ to 15.19$)$ & $(2.86$ to 19.54$)$ & $(1.85$ to 19.54$)$ \\
3D-correction & $4.14 \pm 2.11^{*}$ & $4.23 \pm 2.62^{*}$ & $4.39 \pm 2.49 *$ \\
& $(1.42$ to 8.01$)$ & $(0.98$ to 9.47$)$ & $(0.98$ to 9.47$)$ \\
SI-correction & $3.85 \pm 2.27 *$ & $4.27 \pm 2.68 *$ & $4.13 \pm 2.51^{*}$ \\
& $(1.05$ to 8.75$)$ & $(0.75$ to 9.26$)$ & $(0.75$ to 9.26$)$ \\
\hline
\end{tabular}

BM: Bone matching; DM: diaphragm matching; Head: pancreatic head; Body: pancreatic body; Total: both pancreatic head and body. *There was a significant difference compared to DM.

assumed that our correction method is effective for both regions, because the errors for both parts were smaller than those with BM. However, because there were still errors of $4.13 \pm 2.51 \mathrm{~mm}$ with regard to the tumour position even when using the correction method, caution still needs to be applied.

There are certain limitations to this study. The number of data sets used was insufficient, because there were only 10 patients (14 CT images sets) with data for the pancreatic head and only 17 (18 CT images sets) with data for the pancreatic body. Further analyses with more patient data are necessary. Additionally, positional uncertainty of the DM on X-ray images should be considered, because $\mathrm{DM}$ on the $\mathrm{X}$-ray images taken in clinical practice typically shows larger error than DM performed using CT images. Furthermore, the dose distribution should be confirmed in each position, because this study only examined positional errors.

\section{Conclusion}

We evaluated the effectiveness of DM in carbon-ion radiotherapy for pancreatic cancer, and developed a new method to estimate tumour position. Although there were strong correlations between diaphragm displacements and tumour displacements, DM is not recommended for determining tumour position, because it can lead to larger errors than BM. Our correction method improved the positional errors from the BM and DM.

\section{Conflicts of Interest}

The Authors declare that they have no competing interests in regard to this study.

\section{Funding}

This research did not receive any specific grant from funding agencies in the public, commercial, or not-for-profit sectors.

\section{Authors' Contributions}

YI, YK, and MO coordinated the entire study. Patient clinical data collection was performed by YI, YK, and MO. Data analysis was performed by YI and YK. The article was prepared by YI and YK. Corrections and improvements were suggested by YI, YK, MO, KT, SS, YH, TS, TO, and TN. All Authors read and approved the final article.

\section{Acknowledgements}

The Authors thank the radiology technologists, medical physicists, and medical doctors at GHMC for their valuable insights. We thank Karl Embleton, $\mathrm{PhD}$, from Edanz Group (www.edanzediting.com/ac) for editing a draft of this manuscript.

\section{References}

1 Siegel RL, Miller KD and Jemal A: Cancer statistics, 2019. CA Cancer J Clin 69(1): 7-34, 2019. PMID: 30620402. DOI: 10.3322/caac. 21551

2 Bilimoria KY, Bentrem DJ, Ko CY, Tomlinson JS, Stewart AK, Winchester DP and Talamonti MS: Multimodality therapy for pancreatic cancer in the U.S.A.: utilization, outcomes, and the effect of hospital volume. Cancer 110(6): 1227-1234, 2007. PMID: 17654662. DOI: 10.1002/cncr.22916

3 Kawashiro S, Yamada S, Okamoto M, Ohno T, Nakano T, Shinoto M, Shioyama Y, Nemoto K, Isozaki Y, Tsuji H and Kamada T: Multi-institutional Study of Carbon-ion Radiotherapy for Locally Advanced Pancreatic Cancer: Japan Carbon-ion Radiation Oncology Study Group (J-CROS) Study 1403 Pancreas. Int J Radiat Oncol Biol Phys 101(5): 1212-1221, 2018. PMID: 29907490. DOI: 10.1016/j.ijrobp.2018.04.057

4 Ohno T: Particle radiotherapy with carbon ion beams. EPMA J 4(1): 1-7, 2013. PMID: 23497542. DOI: 10.1186/1878-5085-4-9

5 Irie D, Saitoh JI, Shirai K, Abe T, Kubota Y, Sakai M, Noda SE, Ohno $\mathrm{T}$ and Nakano T: Verification of dose distribution in carbon ion radiation therapy for stage I lung cancer. Int J Radiat Oncol Biol Phys 96(5): 1117-1123, 2016. PMID: 27869084. DOI: 10.1016/j.ijrobp.2016.09.002

6 Sakai M, Kubota Y, Saitoh JI, Irie D, Shirai K, Okada R, Torikoshi M, Ohno T and Nakano T: Robustness of patient positioning for interfractional error in carbon ion radiotherapy for stage I lung cancer: bone matching versus tumor matching. Radiother Oncol 129(1): 95-100, 2018. PMID: 29100701. DOI: 10.1016/j.radonc.2017.10.003

7 Abe S, Kubota Y, Shibuya K, Koyama Y, Abe T, Ohno T and Nakano T: Fiducial marker matching versus vertebral body matching. Dosimetric impact of patient positioning in carbon ion radiotherapy for primary hepatic cancer. Phys Med 33: 114-120, 2017. PMID: 28057427. DOI: 10.1016/j.ejmp.2016.12.018

8 Kubota Y, Katoh H, Shibuya K, Shiba S, Abe S, Sakai M, Yuasa D, Tsuda K, Ohno T and Nakano T: Comparison between bone matching and marker matching for evaluation of intra- and inter- 
fractional changes in accumulated dose of carbon-ion radiotherapy for hepatocellular carcinoma. Radiather Oncol 137: 77-82, 2019. PMID: 31078014. DOI: 10.1016/j.radonc. 2019.04.026

9 Houweling AC, Fukata K, Kubota Y, Shimada H, Rasch CR, Ohno T, Bel A and van der Horst A: The impact of interfractional anatomical changes on the accumulated dose in carbon ion therapy of pancreatic cancer patients. Radiother Oncol 119(2): 319-325, 2016. PMID: 26993417. DOI: 10.1016/ j.radonc.2016.03.004

10 Kubota Y, Tashiro M, Shinohara A, Abe S, Souda S, Okada R, Ishii T, Kanai T, Ohno T and Nakano T: Development of an automatic evaluation method for patient positioning error. J Appl Clin Med Phys 16(4): 100-111, 2015. PMID: 26219004. DOI: 10.1120/jacmp.v16i4.5400

11 Kubota Y, Hayashi H, Abe S, Souda S, Okada R, Ishii T, Tashiro M, Torikoshi M, Kanai T, Ohno T and Nakano T: Evaluation of the accuracy and clinical practicality of a calculation system for patient positional displacement in carbon ion radiotherapy at five sites. J Appl Clin Med Phys 19(2): 144-153, 2018. PMID: 29369463. DOI: $10.1002 / \mathrm{acm} 2.12261$
$12 \mathrm{Li} \mathrm{Y}$, Kubota Y, Tashiro $\mathrm{M}$ and Ohno T: Value of threedimensional imaging systems for image-guided carbon ion radiotherapy. Cancers (Basel) 11(3), 2019. PMID: 30832346. DOI: $10.3390 /$ cancers 11030297

13 Akimoto M, Nakamura M, Nakamura A, Mukumoto N, Kishi T, Goto Y, Mizowaki T and Hiraoka M: Inter- and intrafractional variation in the 3-dimensional positions of pancreatic tumors due to respiration under real-time monitoring. Int J Radiat Oncol Biol Phys 98(5): 1204-1211, 2017. PMID: 28721905. DOI: 10.1016/j.ijrobp.2017.03.042

14 Whitfield G, Jain P, Green M, Henry A, Stratford J, Amer A, Marchant T, Moore C and Price P: Quantifying motion for pancreatic radiotherapy margin calculation. Radiother Oncol 103(3): 360-366, 2012. PMID: 22410203. DOI: 10.1016/ j.radonc.2012.02.012

Received June 6, 2019

Revised June 27, 2019

Accepted June 28, 2019 